Article

\title{
Fourier Transform Infrared Spectroscopy Based Complementary Diagnosis Tool for Autism Spectrum Disorder in Children and Adolescents
}

\author{
Gulce Ogruc Ildiz ${ }^{1,2, *(\mathbb{D} \text {, Sevgi Bayari }}{ }^{3}$, Ahmet Karadag ${ }^{1}$, Ersin Kaygisiz ${ }^{4}\left(\mathbb{D}\right.$ and Rui Fausto ${ }^{2,5}$ (D) \\ 1 Department of Physics, Faculty of Sciences and Letters, Istanbul Kultur University, 34158 Istanbul, Turkey \\ Department of Chemistry, CQC, University of Coimbra, P-3004-535 Coimbra, Portugal; rfausto@ci.uc.pt \\ 3 Department of Physics Engineering, Hacettepe University, 06800 Ankara, Turkey; bayari@hacettepe.edu.tr \\ 4 Department of Geological Engineering, Istanbul University-Cerrahpasa, 34320 Istanbul, Turkey; \\ ersinkygsz@gmail.com \\ 5 Department of Chemistry, King Fahd University of Petroleum and Minerals, Dhahran 34463, Saudi Arabia \\ * Correspondence: g.ogruc@iku.edu.tr
}

Received: 27 February 2020; Accepted: 21 April 2020; Published: 29 April 2020

\begin{abstract}
Autism spectrum disorder (ASD) is a neurodevelopmental disorder that begins early in life and continues lifelong with strong personal and societal implications. It affects about $1 \%-2 \%$ of the children population in the world. The absence of auxiliary methods that can complement the clinical evaluation of ASD increases the probability of false identification of the disorder, especially in the case of very young children. In this study, analytical models for auxiliary diagnosis of ASD in children and adolescents, based on the analysis of patients' blood serum ATR-FTIR (Attenuated Total Reflectance-Fourier Transform Infrared) spectra, were developed. The models use chemometrics (either Principal Component Analysis (PCA) or Partial Least Squares Discriminant Analysis (PLS-DA)) methods, with the infrared spectra being the $X$-predictor variables. The two developed models exhibit excellent classification performance for samples of ASD individuals vs. healthy controls. Interestingly, the simplest, unsupervised PCA-based model results to have a global performance identical to the more demanding, supervised (PLS-DA)-based model. The developed PCA-based model thus appears as the more economical alternative one for use in the clinical environment. Hierarchical clustering analysis performed on the full set of samples was also successful in discriminating the two groups.
\end{abstract}

Keywords: autism spectrum disorder; FTIR spectroscopy; chemometrics

\section{Introduction}

Autism spectrum disorder (ASD) is a neurodevelopmental disorder that begins early in life and continues lifelong. It affects about $1 \%-2 \%$ of the children population in the world. Metabolic diseases, as well as genetic, toxic and environmental factors are recognized as causes of ASD. Symptoms mainly appear as difficulties in social interaction and communication, as well as limited and repetitive patterns of behavior [1-6].

Current diagnosis of ASD is based only on the clinical evaluation of the behavioral signs and symptoms. The absence of auxiliary methods that can complement the clinical evaluation increases the probability of false identification of the disorder, especially in the case of very young children. On the other hand, studies have shown that ASD should be detected at the earliest possible age for the treatment to be effective $[7,8]$.

There have been many attempts to find ASD biomarkers in genetics, neuroimaging, gene expression, and measures of the body's metabolism. Body fluids are easy to collect, and their analysis is less 
expensive compared to neuroimaging and genome studies. Recent studies show that blood samples are one of the most promising targets to search for characteristic biomarkers for ASD, not only due to their easy accessibility but also because of their important biological information content on the health status of children with this disorder [1,4,9-17].

Many studies have been reported aiming to contribute to a better understanding of the underlying causes of ASD. Nevertheless, no biomarkers have yet been identified that can support the clinical diagnosis. Spectroscopic methods, in particular FTIR (Fourier Transform Infrared) spectroscopy, complemented with multivariate methods such as PCA (Principal Component Analysis) and PLS-DA (Partial Least Squares Discriminant Analysis), are becoming powerful tools for the analysis of biological samples, especially body fluids [18-24]. This approach joins the possibility offered by spectroscopy of using molecular information contained in the spectral data, with the analytical efficiency of multivariate statistical methods to process that information. As a whole, the method is cheap, essentially non-destructive and it can be easily implemented in clinical environment. FTIR spectroscopy on blood samples has been used for the investigation of various diseases, including Parkinson's and Alzheimer's, but also different types of cancer and infections, among many others [19,21,23,25-28]. In the present study, we developed chemometrics models based on FTIR data, which can be used as complementary diagnostic tools of ASD in children and adolescents. Besides, specific spectral regions were identified that can act as biomarkers to help distinguishing autistic from healthy individuals.

\section{Materials and Methods}

\subsection{Clinical Stage}

\subsubsection{Patients and Control Group Selection}

A total of 60 children and adolescents ( 30 confirmed ASD cases and 30 controls; Table 1) participated in this study, after consents were obtained from their parents. The ASD group members were chosen among patients that are under treatment in the Child and Adolescent Psychiatry outpatient Clinic of the Pamukkale University (Denizli, Turkey). The group consisted of 23 boys and 7 girls within the age 4-17, diagnosed with ASD according to Diagnostic and Statistical Manual of Mental Disorders [2] criteria. In addition to the diagnostic evaluation, the Childhood Autism Rating Scale (CARS) [29] was applied to evaluate the severity level of the disease. Individuals with other psychiatric disorder and those having a chronic medical comorbid condition were excluded from the study. The healthy control included 22 boys and 8 girls, aged 6 to 16, without any medical or psychiatric history. The study has been approved by the Ethics Committee of Pamukkale University, Faculty of Medicine (date: 12/05/2015; number: 07).

\subsubsection{Samples Preparation}

Five milliliters of venous blood was taken from the antecubital vein of all participants in the study. The collected blood samples were allowed to clot and then centrifuged for $15 \mathrm{~min}$ at $1000 \mathrm{rpm}$ in order to separate the serum from cellular material. The obtained serum samples were aliquoted and stored at $-20^{\circ} \mathrm{C}$ until the analysis.

\subsection{Spectroscopic Stage}

\subsubsection{Sample Measurements}

ATR-FTIR spectra were recorded on a Perkin Elmer Spectrum One spectrometer equipped with a KBr beam splitter and a deuterated triglycine sulfate (DTGS) detector, combined with a diamond GladiATR accessory (Pike Technologies). Sixty-four scans, covering the $4000-450 \mathrm{~cm}^{-1}$ wavenumber range, were co-added to produce each spectrum. A spectral resolution of $4 \mathrm{~cm}^{-1}$ was used. For each blood serum sample, 5 spectra were obtained. 
Before collecting each spectrum, the ATR crystal was first cleaned using sterile phosphate buffer followed by ethanol. Background was collected prior to each sample measurement. For the spectra collection, $1 \mu \mathrm{L}$ of unfrozen blood serum samples were placed on the crystal surface and allowed to air dry ( 12 $\mathrm{min})$ at room temperature.

Table 1. Age and gender distribution in the samples, according to group (ASD or control) ${ }^{a}$.

\begin{tabular}{cccccccccccc}
\hline ASD & Age & Sex & ASD & Age & Sex & Control & Age & Sex & Control & Age & Sex \\
\hline A1 & 10 & B & A16 & 9 & B & C1 & 13 & B & C16 & 10 & B \\
A2 & 9 & B & A17 & 7 & B & C2 & 8 & G & C17 & 11 & B \\
A3 & 6 & B & A18 & 4 & G & C3 & 16 & B & C18 & 10 & B \\
A4 & 7 & G & A19 & 8 & B & C4 & 9 & B & C19 & 8 & B \\
A5 & 12 & B & A20 & 5 & B & C5 & 7 & G & C20 & 8 & B \\
A6 & 14 & B & A21 & 5 & B & C6 & 11 & B & C21 & 16 & B \\
A7 & 4 & G & A22 & 5 & B & C7 & 12 & B & C22 & 10 & B \\
A8 & 10 & B & A23 & 7 & B & C8 & 9 & G & C23 & 10 & B \\
A9 & 14 & G & A24 & 17 & B & C9 & 6 & G & C24 & 11 & B \\
A10 & 5 & B & A25 & 7 & B & C10 & 14 & B & C25 & 8 & B \\
A11 & 7 & B & A26 & 7 & B & C11 & 9 & G & C26 & 12 & B \\
A12 & 6 & B & A27 & 13 & G & C12 & 13 & G & C27 & 8 & G \\
A13 & 17 & B & A28 & 8 & G & C13 & 16 & B & C28 & 8 & B \\
A14 & 14 & B & A29 & 4 & B & C14 & 12 & B & C29 & 10 & B \\
A15 & 5 & B & A30 & 13 & G & C15 & 8 & B & C30 & 7 & G \\
\hline
\end{tabular}

a B, Boy; G, Girl. The first 15 members of the ASD group (A1-A15) and the 14 first members of the control group (C1-C14) were used to develop the models (calibration set); A16-A30 and C15-C29 (15 members of each group) were used to test the models; $\mathrm{C} 30$ was initially included in the calibration set, but was removed since it appeared as an outlier (see below). The numbering in the table was defined after the randomized split of the members of each group between the two sets (calibration and testing sets) and the preliminary investigation to exclude possible outliers.

\subsubsection{Data Pre-processing}

Before analysis, the FTIR spectra were pre-processed by performing baseline correction, and area normalization. No smoothing or any other additional pre-processing of the spectra was performed. For the analyses, the 3700-2400 and 1800-900 $\mathrm{cm}^{-1}$ spectral regions were chosen. The full set of spectra belonging to the totality of samples of the control (C) or ASD (A) groups $(5 \times 30$ spectra for each group) were then subjected to PCA, using the Nonlinear Iterative Partial Least Squares (NIPALS) algorithm [30], in order to detect outliers. This procedure resulted in the elimination of 5 replicas in total, all belonging to the same sample of the control group (C30 sample), which was excluded from the dataset. The average spectrum for each sample was then obtained, as well as the global mean-spectrum for each group ( $\mathrm{C}$ and $\mathrm{A})$.

All data pre-processing was undertaken with the Unscrambler ${ }^{\mathrm{TM}}$ CAMO software (Version 10.5) [31].

\subsubsection{Classification Models Development and Testing}

The dataset used to develop and test the classification models included a total of 59 samples, 30 belonging to the ASD group (A) and 29 to the control group (C). The calibration set comprehended 29 samples (15 for the A group and 14 for the $C$ group), while the test set was formed by 15 samples of each group, in a total of 30 samples. The samples used in the calibration and test sets were chosen randomly.

Two models were built for classification of the samples, one using the PCA method and the other the PLS-DA method [32]. For both models, internal full cross-validation was used during calibration. For predictions, all samples in the test set were used with the two developed models. The hierarchical clustering technique was also applied to the full set of samples, as a preliminary unsupervised test to check the similarity of the samples within each group and the dissimilarity between the two groups. 
The performed cluster analysis used the Ward's method with squared Euclidean distances [33,34]. All chemometric analyses were done using the Unscrambler ${ }^{\mathrm{TM}}$ CAMO software (Version 10.5) [31].

The prediction performance of the models was checked by calculating their sensitivity, specificity, precision, accuracy, and efficiency $[35,36])$. Sensitivity and specificity measure the ability of the model to correctly classify each class and to correctly identify the samples that do not belong to the modelled class, respectively, and are calculated according to: Sensitivity $(\%)=100 \times \mathrm{tp} /(\mathrm{tp}+\mathrm{fn})$; Specificity $(\%)=100$ $x \operatorname{tn} /(\operatorname{tn}+\mathrm{fp})$, where $\mathrm{fp}$ and $\mathrm{fn}$ stand for false positive and false negative samples, respectively, and $\mathrm{tp}$ and th stands for true positive and true negative samples. Precision $(\%)=100 \times \mathrm{tp} /(\mathrm{tp}+\mathrm{fp})$, measures of the quality of the positive predictions of the model. Efficiency and accuracy provide a single measure of the model performance, with efficiency combining the information given by the sensitivity and specificity analyses [efficiency $(\%)=100 \times($ sensitivity + specificity $) / 2$ ], and accuracy measuring the proportion of correct classifications independent of the class [accuracy $(\%)=100$ $\times$ (correct classifications) / total samples].

\section{Results and Discussion}

\subsection{Preliminary Data Analysis}

Figure 1 shows the average IR spectra (area normalized) of the blood serum of the ASD and control groups. Table 2 presents the assignment of the bands, according to the literature [37-43]. The data seems to indicate that the blood serum of the ASD patients have an increase of protein total contents and a slight decrease of tyrosine compared to the control group, while the lipids total amount seems to be nearly identical. Though being only indicative, these results agree with the conclusions of Croonenberghs et al. [44], who reported an increased level of the total protein contents in the blood serum of children with ASD, Elbaz and co-workers [10], Tu, Chen and He [45] and Tirouvanziam et al. [46], who found a significant reduction in tyrosine, and Wiest and co-workers [47], who concluded that the lipid contents in the blood plasma of ASD children and the general population are identical. Besides, the dietary study by Levy et al. [48] also indicated that the protein intake is incremented in ASD children patients.

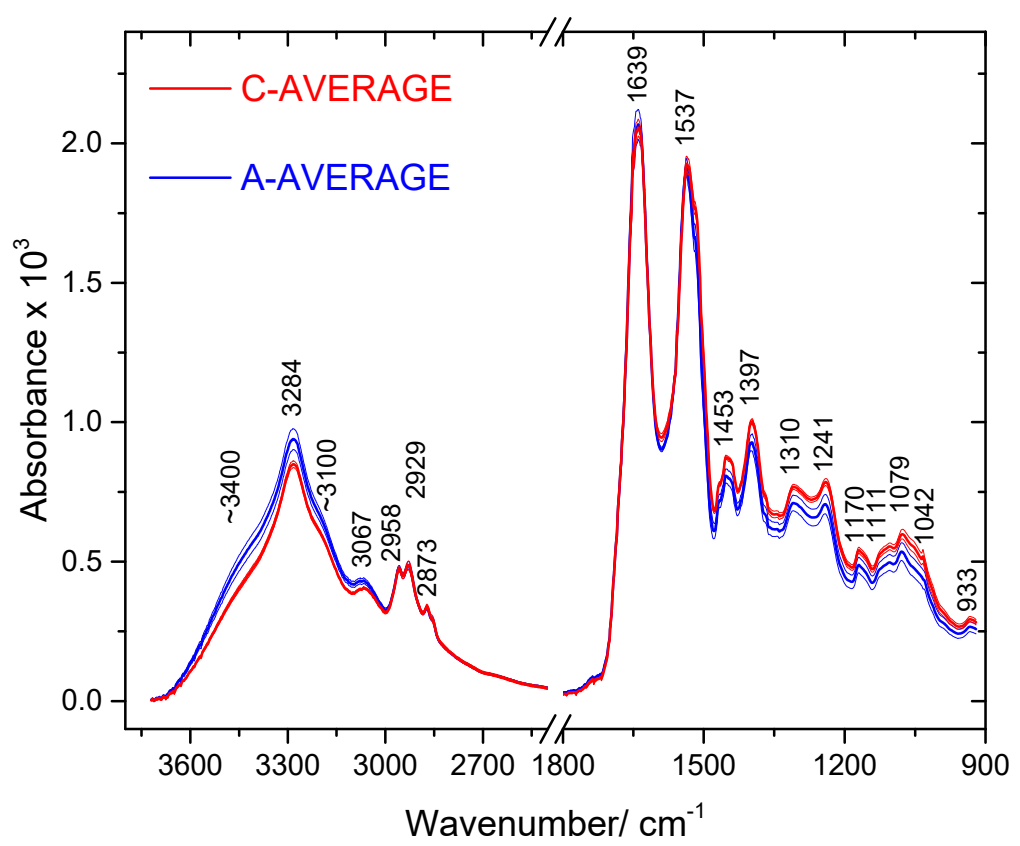

Figure 1. Average IR spectra of ASD (A-AVERAGE; thick blue line) and control (C-AVERAGE; thick red line) groups' blood serum samples (3700-2400 and $1800-900 \mathrm{~cm}^{-1}$ regions). Thin lines account for the standard deviations. 
Figure 2 presents the results of hierarchical clustering analysis performed on the full set of samples, which was undertaken as a preliminary unsupervised similarity test. The samples belonging to the two groups appear clearly discriminated. Noteworthy, the dendrogram also clearly shows that the homogeneity within the control group is significantly higher compared to the ASD group, as expected considering that ASD represents a range of mental disorders of the neurodevelopmental type with different levels of severity (these include disorders previously classified separately and designated as autism and childhood disintegrative disorders, Asperger's syndrome, and pervasive developmental disorder not otherwise specified (PDD-NOS) [2]).

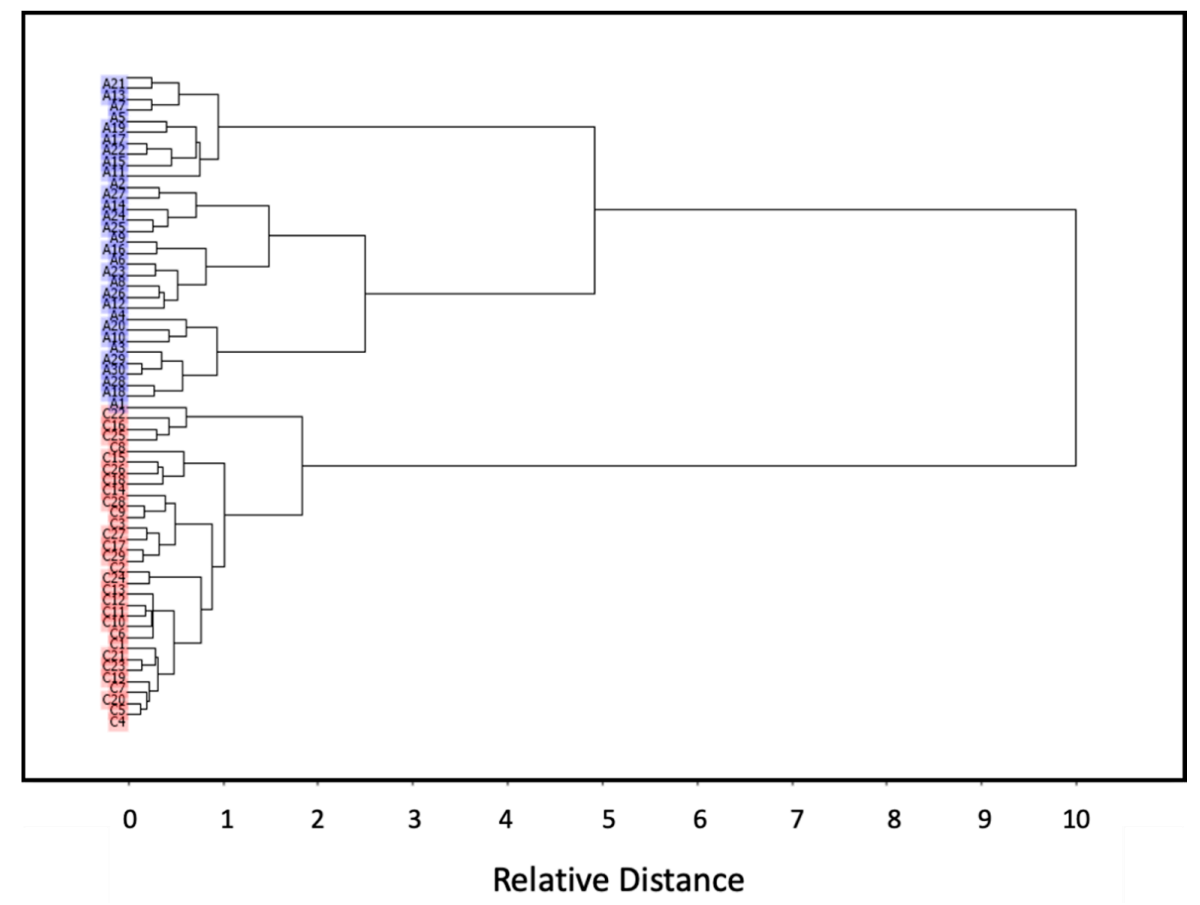

Figure 2. Cluster analysis of ASD (A; overlined in blue) and control (C; highlighted using the red color) groups' blood serum IR spectra, according to the Ward's method, using squared Euclidean distances.

Table 2. Assignments for the major bands in the FTIR spectrum of blood serum ${ }^{a}$.

\begin{tabular}{|c|c|c|c|}
\hline $\begin{array}{c}\text { Wave } \\
\text { Number }\end{array}$ & Assignment & $\begin{array}{c}\text { Wave } \\
\text { Number }\end{array}$ & Assignment \\
\hline$\sim 3400$ & $v(\mathrm{O}-\mathrm{H})$ & 1537 & Amide II (proteins) $\delta(\mathrm{N}-\mathrm{H}) / v(\mathrm{C}-\mathrm{N})$ \\
\hline 3284 & Amide A (proteins) $v(\mathrm{~N}-\mathrm{H})^{b}$ & 1453 & proteins/AA, lipids $\delta_{a s}\left(\mathrm{CH}_{3}\right) \delta\left(\mathrm{CH}_{2}\right)$ \\
\hline$\sim 3100$ & & 1397 & proteins/AA, lipids $\delta_{s}\left(\mathrm{CH}_{3}\right) \mathrm{w}\left(\mathrm{CH}_{2}\right)$ \\
\hline 3067 & Amide B (proteins) $v(\mathrm{~N}-\mathrm{H})^{c}$ & 1310 & Amide III (proteins) $\delta(\mathrm{N}-\mathrm{H}) / v(\mathrm{C}-\mathrm{N})$ \\
\hline 2958 & proteins/AA, lipids vas $\left(\mathrm{CH}_{3}\right)$ & 1241 & nucleic acids vas $\left(\mathrm{PO}_{2}^{-}\right)$ \\
\hline 2929 & proteins/AA, lipids vas $\left(\mathrm{CH}_{2}\right)$ & 1170 & glycogen, nucleic acids, tyrosine $v(\mathrm{C}-\mathrm{O})$ \\
\hline 2873 & proteins, lipids $v_{\mathrm{s}}\left(\mathrm{CH}_{3}\right) v_{\mathrm{s}}\left(\mathrm{CH}_{2}\right)$ & $1111 / 1079 /$ & nucleic acids $v_{s}\left(\mathrm{PO}_{2}^{-}\right)$ \\
\hline 1739 & esters, phospholipids $v(\mathrm{C}=\mathrm{O})$ & 1042 & \\
\hline 1639 & Amide I (proteins) $v(\mathrm{C}=\mathrm{O})$ & 933 & proteins/AA, lipids $\gamma\left(\mathrm{CH}_{2}\right)$ \\
\hline
\end{tabular}

${ }^{a}$ The assignments are according to the literature [37-43]. Wavenumbers in $\mathrm{cm}^{-1}$. AA, amino acids; $\gamma$, stretching; $\delta$, bending; $\mathrm{w}$, wagging; $\gamma$, rocking; s, symmetric; as, anti-symmetric. Bold style in the assignment columns indicate the expected major contributor to the band. In the case of Amide II and III, the main coordinates contributing to the mode are indicated; the first mode corresponds to the anti-phase combination of these coordinates, while the second corresponds to the in-phase combination. ${ }^{b}$ The high-wavenumber wing of the Amide A band is superimposed with the band originated in $\mathrm{OH}$ stretching vibrations, including those due to traces of water still present in the sample. ${ }^{c}$ The Amide B band is partially due to $\mathrm{N}-\mathrm{H}$ stretching vibrations of amide groups involved in strong intramolecular H-bonds, and partially a result of a Fermi resonance interaction between $v \mathrm{NH}$ and the first overtone of the Amide II vibration. 


\subsection{Classification Models Development}

As mentioned before, two models were built for classification of the samples, one based on the PCA method (PCAModel) and the other on the PLS-DA method [32] (PLSModel). For both models the calibration set included the same 29 samples (15 for the A group and 14 for the C group), and internal full cross-validation was used.

Figure 3 shows the PCAModel 2D-scores plot (PC-2 vs. PC-1), where it can be seen that the ASD samples are well discriminated from those belonging to the control group along PC-1. Together, PC-1 and PC-2 explain 98\% of the data variation for the training set (92\% and 6\% variance for PC-1 and PC-2 respectively), with the same numbers for validation. The model was developed using five principal components, accounting for a total variance of 99\% for the training set (validation: $98 \%$ ).

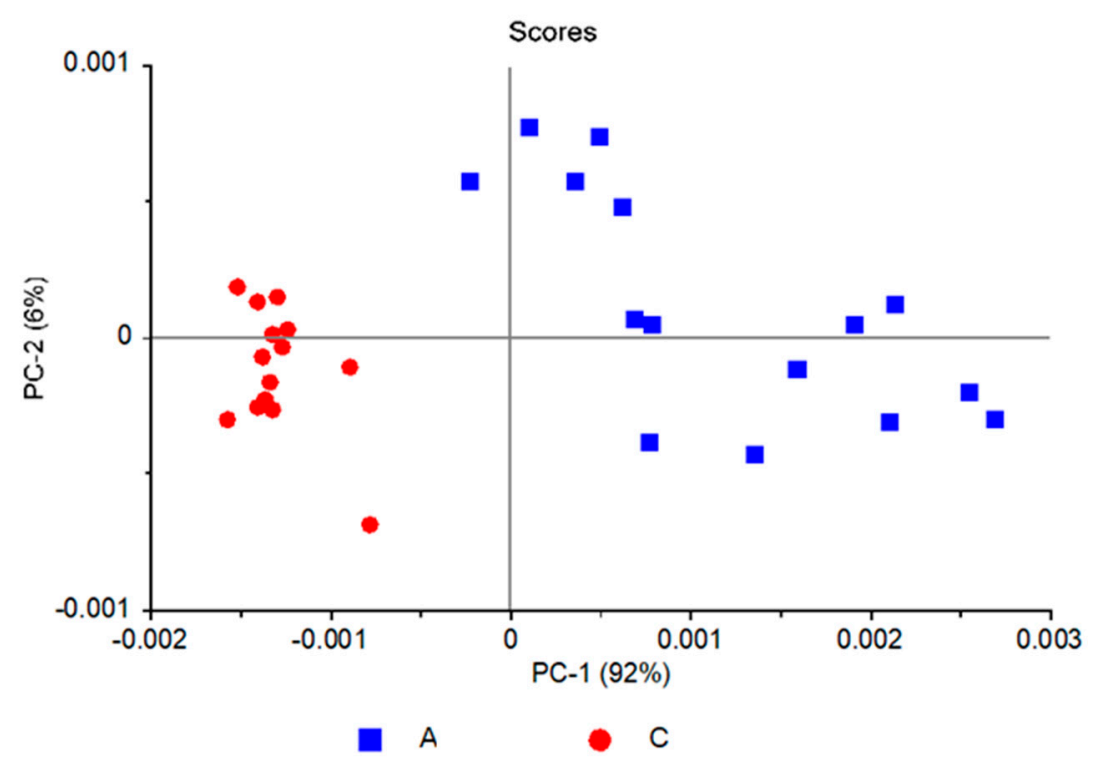

Figure 3. PCA scores plot (PC-2 vs. PC-1) for the PCAModel.

The loadings for PC-1 are given in Figure 4, where they are compared with the difference between the IR spectrum obtained by subtracting the average spectrum of the ASD group from the average spectrum of the control group (see Figure 1 for original spectra). The similarity between the data allows to assign a clear meaning to PC-1. In addition, this similarity can also be correlated with the circumstance that a large amount of variation in the dataset (92\%) is explained by the first principal component. The fact that PC-1 loadings are very much similar to the difference between the average spectra of the two groups (control and ASD) is also relevant because it clearly demonstrates that the achieved discrimination is doubtlessly related with the different nature of the samples. Furthermore, it also validates our main approach to the problem under study, which is the statement that the whole IR spectrum acts as a holistic fingerprint (or spectroscopic biomarker) of the disease, since the PC-1 loadings have significant values for practically all variables (frequency values).

It is also interesting to note that the samples distribution within each group along PC-1 is substantially different, with the samples belonging to the control group spawning along a small range of values and those belonging to the ASD group (A) spreading a much wider range. This result reflects the greater homogeneity of the samples in the control group $(C)$ compared to the ASD group, thus closely following the trend observed in the hierarchical clustering analysis discussed in the previous section. Along PC-2 the distribution of samples does not differ very much from one group to the other, indicating that the variance explained by this principal component reflects general small differences in the spectra that are not related with the different nature of the two groups (A vs. C). 

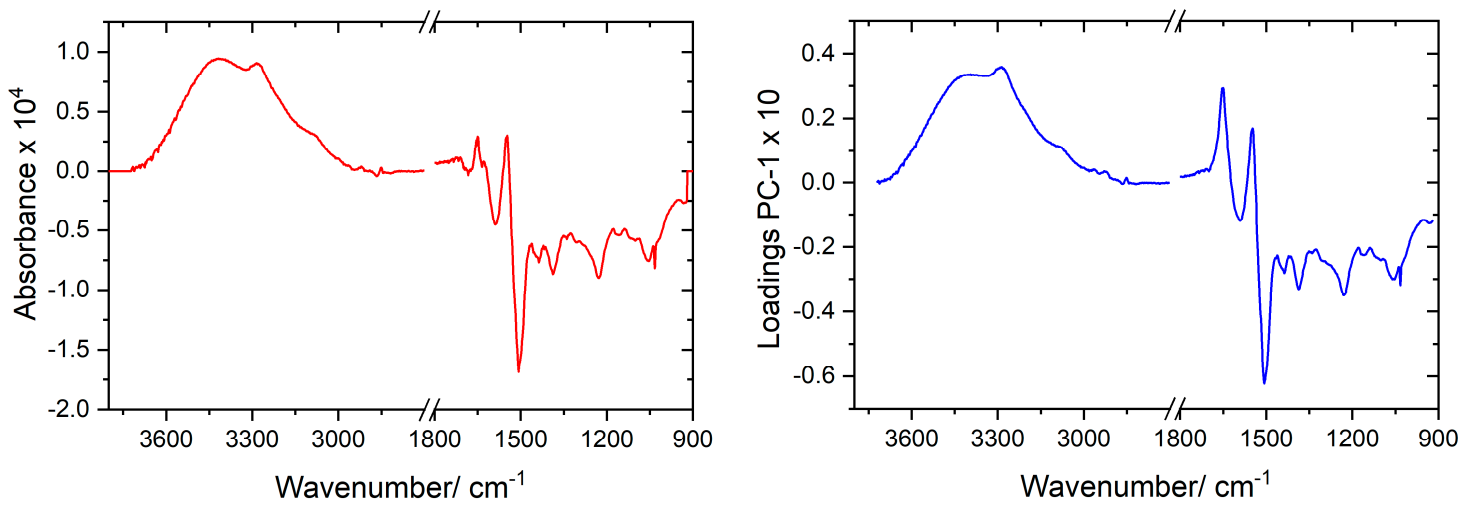

Figure 4. Left panel: Difference IR spectrum (average spectrum of ASD group (A) blood serum minus average spectrum of the control group (C) blood serum). Right panel: PC-1 loadings of PCAModel.

The 2D-scores plot (Factor-2 vs. Factor-1) for the PLSModel is shown in Figure 5. As it could be expected considering the results obtained using the unsupervised PCA-based model (PCAModel) described above, the supervised PLSModel discriminates well the ASD samples from the control ones. The results obtained with the two models are in fact very similar. In the PLSModel, in parallel to what was found for the PCAModel, the groups are discriminated along the axis explaining the largest fraction of variance (Factor-1, whose loadings are also identical to the difference between the average IR spectra of the control and ASD groups, like the PC-1 loadings in the PCAModel), while along Factor-2 the distribution of samples are rather identical. Additionally, as it was found for the PCAModel, along Factor-1 the samples belonging to the ASD group are dispersed along a much wider range of score values than those belonging to the control group. The reasons for the two last trends, common to the two developed models, have already been provided above.

In the PLSModel, the two latent variables explaining the largest amounts of variation (Factor- 1 and Factor-2) account for $97 \%$ of the variation in $X$ and $90 \%$ in $Y$ for the training set $(91 \%$ and $80 \%$ variance in $X$ and $Y$, respectively, for Factor- 1 , and $6 \%$ and 10\% for Factor-2), with similar numbers observed for validation (91\% and 5\% variance in $X$, and $78 \%$ and $12 \%$ variance in $Y$ for Factor- 1 and -2 respectively; the totals were $96 \%$ and $90 \%$ for $X$ and $Y$ variance, respectively). The model was developed using five latent variables, accounting for total $X$ and $Y$ variances of $99 \%$ and $98 \%$ for the training set (validation: $98 \%$ and 93\%). The root-mean-square errors (RMSE) for training and validation are 0.10 and 0.14 , respectively, which demonstrate the good quality of the regressions.

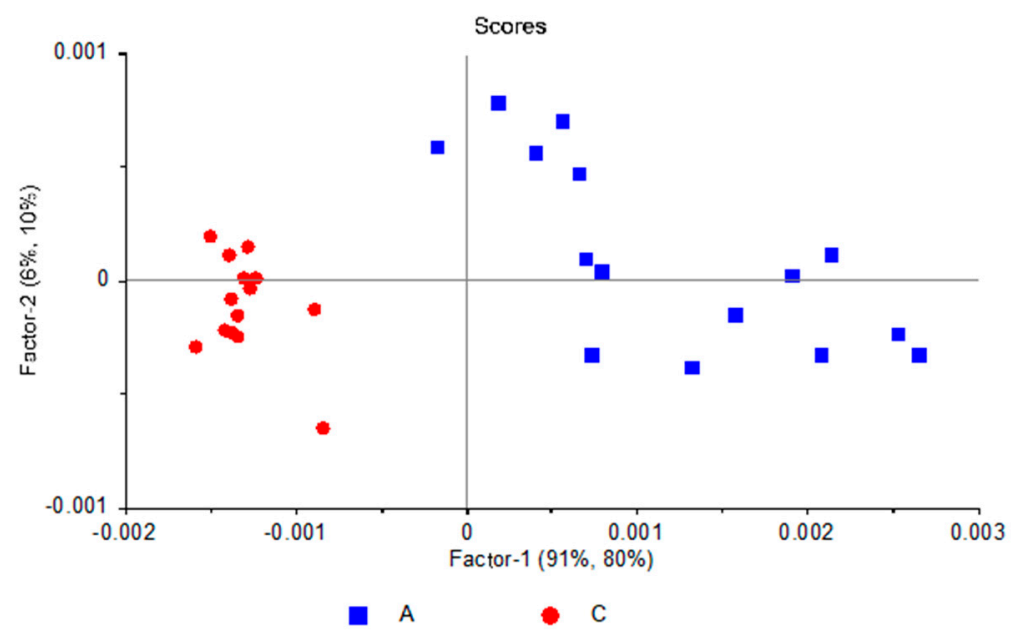

Figure 5. Scores plot (Factor-2 vs. Factor-1) for the PLSModel. 


\subsection{Predictions}

Fifteen samples of each group (A and C) not used for calibration of the models were used as test set for predictions. The IR spectra of the test samples were pre-processed following the same steps as for the samples used in the calibration of the models. The results obtained for the predictions done using the two models are summarized in Figures 6-8.

Both models were able to classify correctly all samples included in the test set, with no false positives or negatives (global accuracy, 100\%), so that the calculated values for all parameters chosen to measure the prediction performance of the models (sensitivity, specificity, precision, accuracy, and efficiency [35,36], whose meaning was given in Section 2.2.3) are maximal (100\%). Figures 6 and 7 show the projections of the test samples on the scores plots of the models, while Figure 8 gives the PLSModel predicted $Y$ values for the samples and deviations, the last being an indicator of how reliable the predicted values are [49].

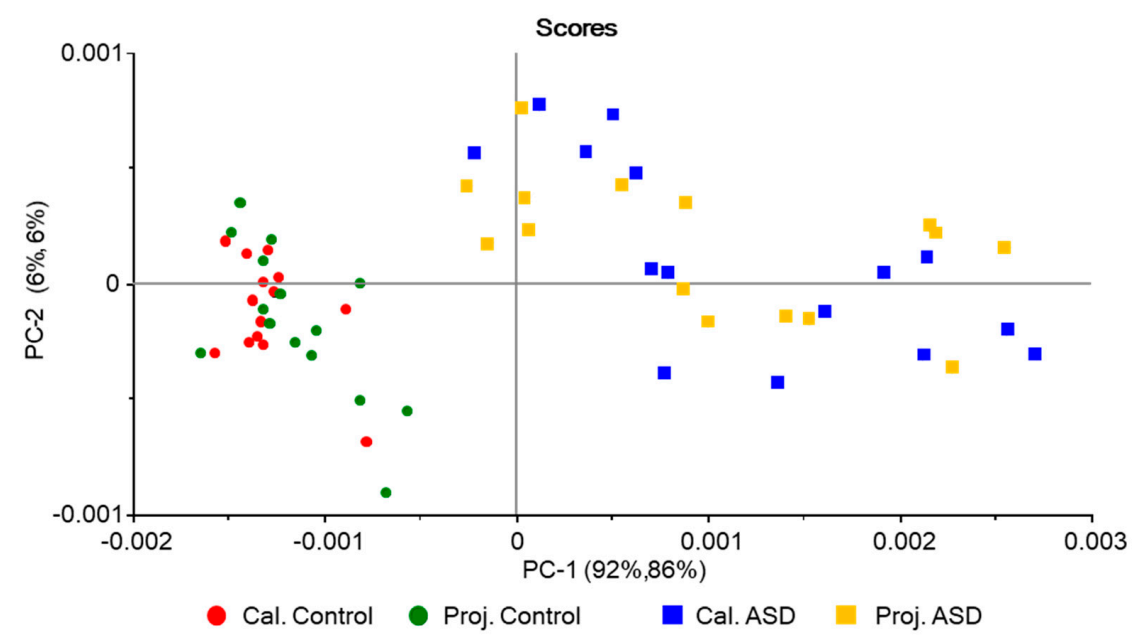

Figure 6. Projection scores plot (PC2 vs. PC-1) for the PCAModel.

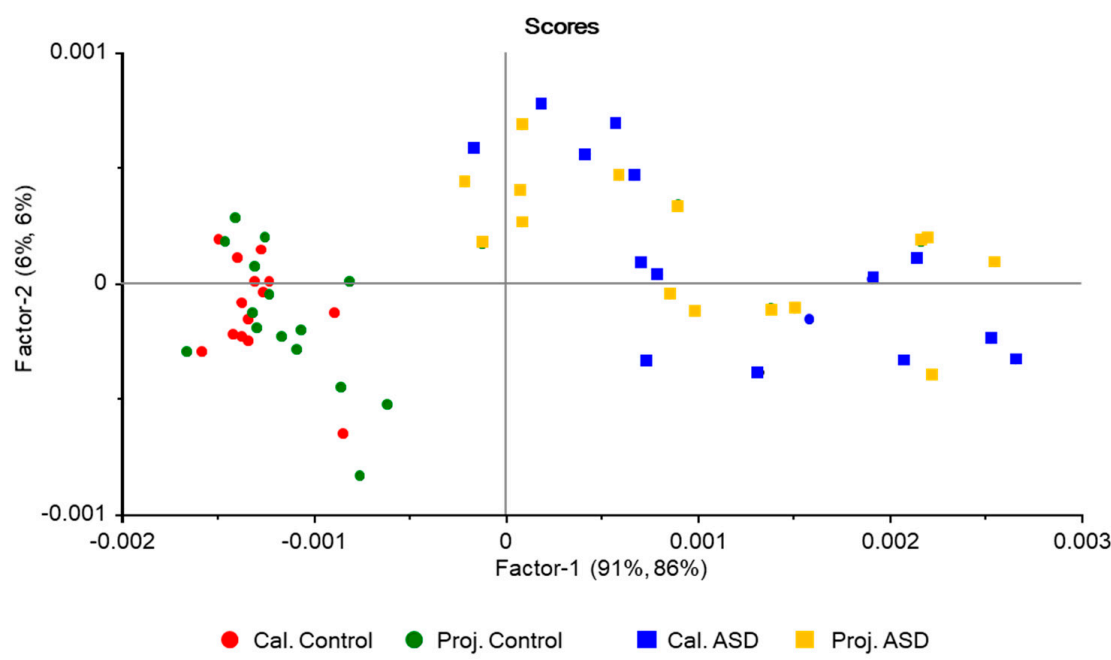

Figure 7. Projection scores plot (Factor-2 vs. Factor-1) for the PLSModel.

For the PCAModel, the predicted class for the samples was assigned based on the inspection of their projection on the model scores plot (Figure 6). The samples were ascribed as belonging to the same group to which the samples corresponding to its nearest three neighbor points belong. In the case of the PLSModel, the class assignments were done by considering the predicted $Y$ values for the samples, 
using as threshold value for class separation the half distance between the reference $Y$ values (0.5). It shall be noticed that the predicted $Y$ values for the ASD test samples show a larger dispersion around the reference value compared to the control test samples. This result is in consonance with the already mentioned smaller homogeneity between the samples belonging to the ASD patients in comparison with those of the control group.

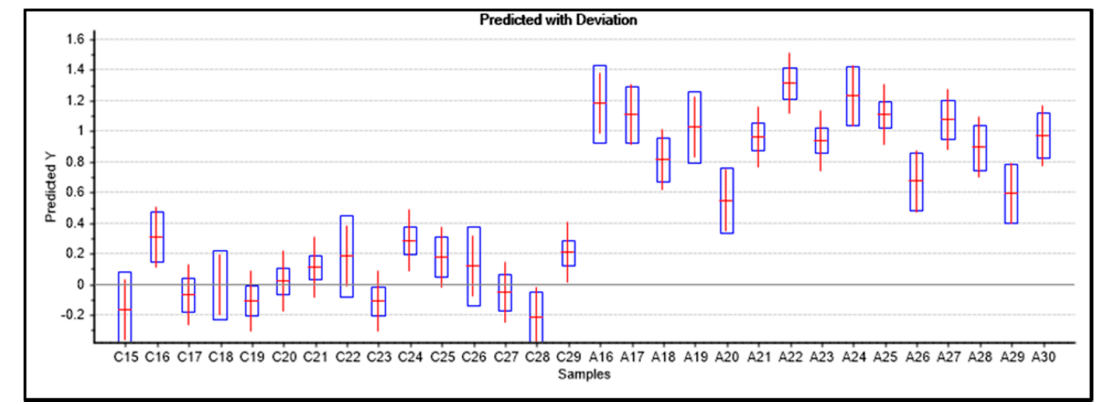

Figure 8. PLSModel predicted $Y$ values for ASD (A group) and control (C group) test samples. The predicted values are indicated by the horizontal red lines, and the deviations by the blue boxes. In the model, samples belonging to control group define class 1 (value 0 for $Y$ ) and samples belonging to ASD patients define class 2 (value 1 for $Y$ ).

The fact that the two models show a similar prediction performance is striking. In fact, the performance of the PCA-based model is so good that the model derived using the supervised PLS-DA method, which could a priori be expected to outperform the unsupervised PCA-based model, does not improve on this latter in a noticeable way. Under these circumstances, the simplest PCAModel appears to be the most convenient model for practical use.

The PCAModel was also checked in relation to its robustness. For that, we performed an additional PCA calculation using all 59 samples in the training set. The idea was to verify if including a larger number of samples in the training set would visibly change the description of the data by the model. The obtained scores plot is presented in Figure 9. In this PCA PC-1 and PC-2 explain 95\% of the data variation for the training set (89\% and 6\% variance for PC-1 and PC-2 respectively), with equal numbers for validation. The five principal components used in the PCA account for a total variance of $98 \%$ both for training set and validation. Noteworthy, the scores plot of this PCA (Figure 9), with all 59 samples in the calibration set, closely matches the projection graph obtained for the model developed with only 29 samples in the calibration set (see Figure 6), a result that clearly shows the robustness of the PCAModel.

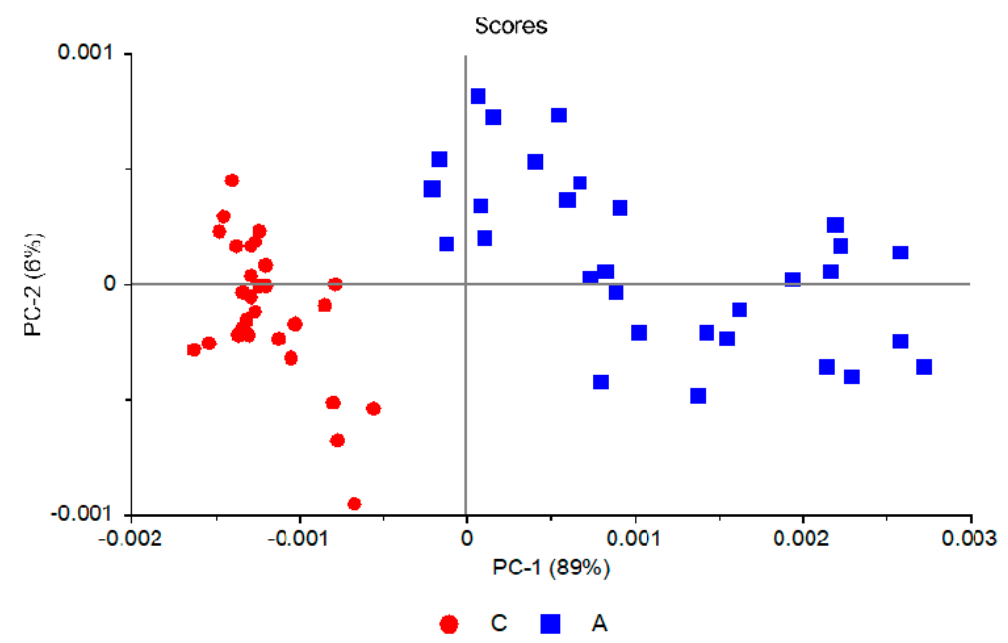

Figure 9. Scores plot (PC-2 vs. PC-1) for the PCA done using all 59 samples. 


\section{Conclusions}

In this study we developed analytical models for auxiliary diagnosis of ASD in children and adolescents, based on the analysis of patients' blood serum infrared spectra. The models use chemometric (either PCA or PLS-DA) methods, with the infrared spectra acting as the X-predictor variables. The two developed models exhibit excellent classification/prediction performance. Remarkably, the simplest, unsupervised PCA-based model results to have a performance identical to the more expensive, supervised (PLS-DA)-based model. The developed PCA-based model thus appears as the best, more economical alternative for use in the clinical environment.

It can be concluded that infrared spectrum of the blood serum can be used for the discrimination of ASD patients from healthy controls. Since it considers the whole spectroscopic information to achieve classification, this approach is conceptually more consistent than the putative alternative ones that aim to use spectroscopic data of complex biological materials to find specific molecular biomarkers for the disease.

The obtained results regarding the relative similarity of the samples within each one of the two studied groups (ASD and control groups), clearly showing the much greater dissimilarity between the samples belonging to the ASD group, are in accordance with the modern psychiatric concept of ASD being a general type of disorder which includes a spectrum of clinical manifestations which previously were considered different psychiatric illnesses.

The methodology proposed herein is reliable, fast, cheap, essentially non-invasive, and might be implemented easily in the clinical environment in order to help psychiatrists to establish with increased certainty the ASD diagnosis at an early stage of development of the illness.

Author Contributions: G.O.I., R.F. and S.B. performed the spectral and chemometric analyses and wrote the original version of the manuscript. A.K. and E.K. carried out the FTIR spectroscopy measurements. All authors contributed to the final form of the study. All authors have read and agreed to the published version of the manuscript

Funding: This research was funded by the Scientific and Technological Research Council of Turkey-TUBITAK 1001 Project, grant number 116Z294 and R.F. thanks the Portuguese Science Foundation (Fundação para a Ciência e a Tecnologia; FCT) for financial support to the Coimbra Chemistry Centre (CQC, Research Unit UI0313/QUI/2020) co-funded by FEDER/COMPETE 2020-EU.

Acknowledgments: The authors thank Med. Drs. Ahmet Buber (Pamukkale University, Faculty of Medicine, Child and Adolescent Psychiatry Department, Denizli, Turkey) and Omer Faruk Tuncer (Bursa Dortcelik Pediatric Hospital, Bursa, Turkey) for their contribution in the clinical stage of the study.

Conflicts of Interest: The authors declare no conflict of interest

\section{References}

1. Zurawicz, E.; Kaluzna-Czaplinska, J. Analysis of amino acids in autism spectrum disorders. Trends Anal. Chem. 2015, 73, 91-118. [CrossRef]

2. American Psychiatric Association. Diagnostic and Statistical Manual of Mental Disorders, Fifth Edition; American Psychiatric Association: Arlington, VA, USA, 2013.

3. Autism and Development Disabilities Monitoring Network Surveillance Year 2008 Principal Investigators and Centers for Disease Control and Prevention. MMWR Surveill. Summ. 2012, 61, 1-19.

4. Ruggeri, B.; Sarkans, U.; Schumann, G.M.; Persico, A. Biomarkers in autism spectrum disorder: The old and the new. Psychopharmacology 2014, 231, 1201-1216. [CrossRef] [PubMed]

5. Wetie, A.G.N.; Wormwood, K.; Thome, J.; Dudley, E.; Taurines, R.; Gerlach, M.; Woods, A.G.; Darie, C.C. A Pilot Proteomic Study of Protein Markers in Autism Spectrum Disorder. Electrophoresis 2014, 35, $2046-2054$. [CrossRef]

6. Abruzzo, P.M.; Ghezzo, A.; Bolotta, A.; Ferreri, C.; Minguzzi, R.; Vignini, A.; Visconti, P.; Marini, M. Perspective Biological Markers for Autism Spectrum Disorders: Advantages of the Use of Receiver Operating Characteristic Curve s in Evaluating Marker Sensitivity and Specificity. Dis. Markers 2015, 329607, 1-15. [CrossRef] 
7. Eikeseth, S.; Klintwall, L.; Jahr, L.E.; Karlsson, P. Outcome for children with autism receiving early and intensive behavioral intervention in mainstream preschool and kindergarten settings. Res. Autism Spectr. Disord. 2012, 6, 829-835. [CrossRef]

8. Eldevik, S.; Hastings, P.R.; Hughes, C.J.; Jahr, E.; Eikeseth, S.; Cross, S. Meta-Analysis of Early Intensive Behavioral Intervention for Children With Autism. J. Clin. Child. Adolesc. Psych. 2009, 38, 439-450. [CrossRef]

9. Goldani, A.A.S.; Downs, S.R.; Widjaja, F.; Lawton, B.; Hendren, R.L. Biomarkers in autism. Front. Psychiatry 2014, 5, 1-13. [CrossRef]

10. ElBaz, F.M.; Zaki, M.M.; Youssef, A.M.; ElDorry, G.F.; Elalfy, D.Y. Study of plasma amino acid levels in children with autism: An Egyptian sample. Egypt. J. Med. Hum. Genet. 2014, 15, 181-186. [CrossRef]

11. Cetin, I.; Tezdig, I.; Tarakcioglu, M.C.; Kadak, M.T.; Demirel, O.F.; Ozer, O.F. Serum levels of glial fibrillary acidic protein and Nogo-A in children with autism spectrum disorders. Biomarkers 2016, 21, 614-618. [CrossRef]

12. West, P.R.; Amaral, D.G.; Bais, P.; Smith, A.M.; Egnash, L.A.; Ross, M.E.; Palmer, J.A.; Fontaine, B.R.; Conard, K.R.; Corbett, B.A.; et al. Metabolomics as a Tool for Discovery of Biomarkers of Autism Spectrum Disorder in the Blood Plasma of Children. PLOS ONE 2014, 9, 1-13. [CrossRef] [PubMed]

13. Wang, L.; Jia, J.; Zhang, J.; Li, K. Serum levels of SOD and risk of autism spectrum disorder: A case-control study. Int. J. Devl. Neurosci. 2016, 51, 12-16. [CrossRef] [PubMed]

14. Jory, J. Abnormal fatty acids in Canadian children with autism. Nutrition 2016, 32, 474-477. [CrossRef]

15. Kondolota, M.; Ozmert, E.N.; Asci, A.; Erkekoglu, P.; Oztop, D.B.; Gumus, H.; Kocer-Gumus, B.; Yurdak, K. Plasma phthalate and bisphenol a levels and oxidant-antioxidant status in autistic children. Env. Toxicol. Pharm. 2016, 43, 149-158. [CrossRef] [PubMed]

16. Smaga, I.; Niedzielska, E.; Gawlik, M.; Moniciewski, A.; Krzek, J.; Przegalinski, E.; Pera, J.; Filip, M. Oxidative stress as an etiological factor and a potential treatment target of psychiatric disorders. Part 2. Depression, anxiety, schizophrenia and autism. Pharm. Rep. 2015, 67, 569-580. [CrossRef] [PubMed]

17. Zaman, S.; Yazdani, U.; Deng, Y.; Li, W.; Gadad, B.S.; Hynan, L.; Karp, D.; Roatch, N.; Schutte, C.; Marti, C.N.; et al. Search for Blood Biomarkers for Autism: Peptoids. Sci. Rep. 2016, 4, 1-8. [CrossRef]

18. Kanagathara, N.; Thirunavukkarasu, M.; Jeyanthi, E.C.; Shenbagarajan, P. FTIR and UV-Visible spectral study on normal blood samples. Int. J. Pharm. Bio. Sci. 2011, 1, 74-81.

19. Ahmed, S.S.S.J.; Santosh, W.; Kumar, S.; Christlet, T.H.T. Neural network algorithm for the early detection of Parkinson's disease from blood plasma by FTIR micro-spectroscopy. Vib. Spectrosc. 2010, 53, 181-188. [CrossRef]

20. Conti, C.; Giorgini, E.; Pieramici, T.; Rubini, C.; Tosi, G. FT-IR microscopy imaging on oral cavity tumours, II. J. Mol. Struct. 2005, 744, 187-193. [CrossRef]

21. Deleris, G.; Petibois, C. Applications of FT-IR spectrometry to plasma contents analysis and monitoring. Vib. Spectrosc. 2003, 32, 129-136. [CrossRef]

22. Perez-Guaita, D.; Sanchez-lllana, A.; Ventura-Gayete, J.; Garrigue, S.; Guardia, M. Chemometric determination of lipidic parameters in serum using ATR measurements of dry films of solvent extracts. Analyst 2014, 139, 170-178. [CrossRef] [PubMed]

23. Khanmohammadi, M.; Ghasemi, K.; Garmarudi, A.B.; Ramin, M. Diagnostic prediction of renal failure from blood serum analysis by FTIR spectrometry and chemometrics. Spectrochim. Acta Part A Mol. Biomol. Spectrosc. 2015, 136, 1782-1785. [CrossRef] [PubMed]

24. Dovbeshko, G.I.; Gridina, N.Y.; Kruglova, E.B.; Pashchuk, O.P. FTIR spectroscopy studies of nucleic acid damage. Talanta 2000, 53, 233-246. [CrossRef]

25. Mostaco-Guidolin, L.B.; Bachmann, L. Application of FTIR Spectroscopy for Identification of Blood and Leukemia Biomarkers: A Review over the Past 15 Years. Appl. Spectrosc. Rev. 2011, 46, 388-404. [CrossRef]

26. Erukhimovitch, V.; Talyshinsky, M.; Souprun, Y.; Huleihel, M. FTIR spectroscopy examination of leukemia patients plasma. Vibrat. Spectrosc. 2006, 40, 40-46. [CrossRef]

27. Lewis, P.D.; Lewis, K.E.; Ghosal, R.; Bayliss, S.; Lloyd, A.J.; Wills, J.; Godfrey, R.; Kloer, P.; Mur, L.A.J. Evaluation of FTIR Spectroscopy as a diagnostic tool for lung cancer using sputum. BMC Cancer 2010, 10, 640. [CrossRef]

28. Mordechai, S.; Shufan, E.; Katz Porat, B.S.; Salman, A. Early diagnosis of Alzheimer's disease using infrared spectroscopy of isolated blood samples followed by multivariate analyses. Analyst 2017, 142, 1276-1284. [CrossRef]

29. Gassaloglu, S.I.; Baykara, B.; Avcil, S.; . Demiral, Y. Validity and Reliability Analysis of Turkish Version of Childhood Autism Rating Scale. Turk Psikiyatr. Derg. 2016, 27, 266-274. 
30. Wold, H. Quantitative Sociology: International Perspectives on Mathematical and Statistical Model Building; Academic Press: New York, NY, USA, 1975; pp. 307-357.

31. CAMO Software Inc. The Unscrambler Version 10.5; CAMO A/S: Trondheim, Norway, 2018.

32. Barker, M.; Rayens, W. Partial Least Squares For Discrimination. J. Chemom. 2003, 17, 166-173. [CrossRef]

33. Ward, J.H.J. Hierarchical Grouping to Optimize an Objective Function. J. Am. Stat. Assoc. 1963, 58, $236-244$. [CrossRef]

34. Murtagh, F.; Legendre, P. Ward's Hierarchical Agglomerative Clustering Method: Which Algorithms Implement Ward's Criterion? J. Classif. 2014, 31, 274-295. [CrossRef]

35. Wiest, M.M.; German, J.B.; Harvey, D.J.; Watkins, S.M.; Hertz-Picciotto, I. Plasma fatty acid profiles in autism: A case-control study. Prostaglandins Leukot. Essent. Fat. Acids 2009, 80, 221-227. [CrossRef] [PubMed]

36. Levy, S.E.; Souders, M.C.; Ittenbach, R.F.; Giarelli, E.; Mulberg, A.E.; Pinto-Martin, J.A. Relationship of dietary intake to gastrointestinal symptoms in children with autistic spectrum disorders. Biol. Psychiatry 2007, 61, 492-497. [CrossRef] [PubMed]

37. Krimm, S.; Bandekar, J. Vibrational spectroscopy and conformation of peptides, polypeptides, and proteins. Adv. Protein Chem. 1986, 38, 181-364.

38. Liu, K.Z.; Shi, M.H.; Mantsch, H.H. Molecular and chemical characterization of blood cells by infrared spectroscopy: A new optical tool in hematology. Blood Cells Mol. Dis. 2005, 35, 404-412. [CrossRef]

39. Krilov, D.; Balarin, M.; Kosovic, M.; Gamulin, O.; Brnjas-Kralkevic, J. FT-IR spectroscopy of lipoproteins-a comparative study. Spectrochim. Acta A 2009, 73, 701-706. [CrossRef]

40. Petibois, C.; Rigalleau, V.; Melin, A.M.; Perromat, A.; Cazorla, G.; Gin, H.; Deleris, G. Determination of glucose in dried serum samples by Fourier-transform infrared spectroscopy. Clin. Chem. 1999, 45, 1530-1535. [CrossRef]

41. Paraskevaidi, M.; Morais, C.L.M.; Lima, K.M.G.; Snowden, J.S.; Saxon, J.A.; Richardson, A.M.T.; Jones, M.; Mann, D.M.A.; Allsop, D.; Martin-Hirsch, P.L.; et al. Differential diagnosis of Alzheimer's disease using spectrochemical analysis of blood. Proc. Natl. Acad. Sci. USA 2017, 2017, E7929-E7938. [CrossRef]

42. Ramalingam, P.; Reddy, P.; Kumar, K.V.; Candu, B.R.; Rajendran, K. Evaluation of metformin hydrochloride in Wistar rats by FTIR-ATR spectroscopy: A convenient tool in the clinical study of diabetes. J. Nat. Sci. Biol. Med. 2014, 5, 288-292. [CrossRef]

43. Sheng, D.; Liu, X.; Li, W.; Yang, Y.; Chen, X.; Wang, X. Distinction of leukemia patients' and healthy persons' serum using FTIR spectroscopy. Spectrochim. Acta Part A Mol. Biomol. Spectrosc. 2013, 101, 228-232. [CrossRef]

44. Croonenberghs, J.; Bosmans, E.; Deboutte, D.; Kenis, G.; Maes, M. Activation of the Inflammatory Response System in Autism. Neuropsychobiology 2002, 45, 1-6. [CrossRef]

45. Tu, W.J.; Chen, H.; He, J. Application of LC-MS/M analysis of plasma amino acids profiles in children with Autism. J. Clin. Biochem. Nutr. 2012, 5, 1248-1249. [CrossRef] [PubMed]

46. Tirouvanziam, R.; Obukhanych, T.V.; Laval, J.; Aronov, P.A.; Libove, R.; Banerjee, A.G.; Parker, K.J.; Ohara, R.; Herzenberg, L.A.; Herzenberg, L.A.; et al. Distinct Plasma Profile of Polar Neutral Amino Acids, Leucine, and Glutamate in Children with Autism Spectrum Disorders. J. Autism Dev. Disord. 2011, 42, 827-836. [CrossRef] [PubMed]

47. Chao, L.; Mei, H.; Pan, X.C.; Tan, W.; Liu, T.F.; Yang, L. An improved large-scale prediction model of CYP1A2 inhibitors by using combined fragment descriptors. Chemom. Intell. Lab. Syst. 2014, 130, 109-114. [CrossRef]

48. Campos, N.D.S.; Oliveira, K.S.; Almeida, M.R.; Stephan, R.; Oliveira, L.F.C. Classification of Frankfurters by FT-Raman Spectroscopy and Chemometric Methods. Molecules 2014, 19, 18980-18992. [CrossRef]

49. De Vries, S.; Ter Braak, C.J.F. Prediction error in partial least squares regression: A critique on the deviation used in The Unscrambler. Chemom. Intell. Lab. Syst. 1995, 30, 239-245. [CrossRef]

Sample Availability: Samples of the compounds are not available from the authors. 